\title{
Literatur
}

1. Boyd, J. W., Biochem. J. 81, 434 (1961). - 2. Boyd, J. W., Clin. chim. Acta (Amsterdam) 7, 424 (1962). - 3. Decker, L. E. und E. M. RAu, Proc. Soc. exp. Biol. Med. 112, 144 (1963). - 4. Fleisher, G. A., C. S. Potter und K. G. WAkim, Proc. Soc. exp. Biol. Med. 103, 229 (1960). - 5. Fletsher, G. A. und K. G. WAKIM, Proc. Soc. exp. Biol. Med. 106, 283 (1961). - 6. Fleisher, G. A. und K. G. WAkm, J. Laborat. Clin. Med., S. Louis 61, 76 (1963). - 7. Fleisher, G. A. und K. G. Wakim, J. Laborat. Clin. Med., S. Louis 61, 98 (1963). - 8. Schwartz, M. K., J. S. Nisselbaum und O. Bodansky, Amcr. J. Clin. Path. 40, 103 (1963). - 9. Wakim, K. G. und G. A. Fleisher, J. Laborat. Clin. Med., S. Louis, 61, 86 (1963). 10. WAKIM, K. G. und G. A. Fleisiler, J. Laborat. Clin. Med., S. Louis 61107 (1963). - 11. ZizvoRkA, Z. und J. KamarÝt, Čsl. Gastroenterol. 19, 39 (1965). - 12. ZízvorKa, Z. und J. KamarÝt, Čcs. lćk. česk. (im Druck). - 13. ZízvorKa, Z. und J. Kamarút, J. Laborat. Clin. Med., S. Louis (im Druck) - 14. Kamarł́, J. und Z. ZÁZvorkA, Die Geltung der simultanen Agar-Gel-Enzym-Elektrophorese in der präventiven Medizin, in: Ergebnisse der Laboratoriumsmedizin, Bd. II S. 185 MedicusVerlag GMBH, Berlin (1965). - 15. ZÁzvorKA, Z. und J. KaMARYT, LDH, MDH und GOT-Isoenzyme in der Früh-Diagnostik der Leber-, Herz- und Bluterkrankungen; in: Ergebnisse der Laboratoriumsmedizin, Bd. II, S. 41, Medicus-Verlag GMBH, Berlin (1965). - 16. Kellen, J. und V. Romanč́́k, diese Z. (im Druck).

\section{Schnellreaktion auf p-Nitrophenol im Harn zum Nachweis einer E 605-Vergiftung $\left.{ }^{1}\right)$}

\author{
Dr. med. Zd. Zázvorka, \\ Most, Palackého 1837, ČSSR
}

Herrn Prof. Dr. Dr. F. Timm zum 70. Geburtstag gewidmet

Von M. Geldmacher-v. Mallinckrodt und K. Deinzer

Aus dem Institut für gerichtliche Medizin und Kriminalistik der Universität Erlangen-Nürnberg (Direktor: Prof. Dr. Dr. E. Weinig)

(Eingegangen am 23. September 1965)

\begin{abstract}
Der p-Nitrophenol-Nachweis im Harn nach v. EICKen wird als Schnellreaktion zum Nachweis einer E 605-Vergiftung ausgebaut. Die Arbeitszeit wird dabei von 2 Stunden auf 15 Minuten reduziert.

The detection of p-nitrophenol in urine after v. ErCKEN has been developed as a rapid test for E 605 poisoning. The operation time is reduced from 2 hours to 15 minutes.
\end{abstract}

Vergiftungen mit dem Schädlingsbekämpfungsmittel „E 605“ spielen nach wie vor eine große Rolle. Sie haben auf Grund der heutigen therapeutischen Möglichkeiten eine wesentlich bessere Prognose als früher. Voraussetzung ist allerdings die schnell und sicher gestellte Diagnose. Hierfür haben SCHwerd und SCHMIDT schon 1952 eine Schnellreaktion im Blut angegeben, die auf der durch p-Nitrophenol-Na hervorgerufenen Gelbfärbung nach Spaltung des im Serum vorhandenen E 605 beruht (1). Es besteht aber die Gefahr der Täuschung durch eine unspezifische Gelbfärbung $(2,3)$.

Sicherer und spezifischer erscheint der Nachweis von pNitrophenol im Harn. Schon 1951 haben MountarN, Zlorolow und O'Conor darauf hingewiesen, daß im Organismus als Spaltprodukt des E 605 das p-Nitrophenol auftritt und mit der Indophenolblau-Reaktion im Urin nachweisbar ist (4). O'BRIEN (5) gibt ein Stoffwechselschema für die Kuh an, das alle bekannten Umwandlungen des E 605-Moleküls im Organismus enthält und das wohl allgemein für Warmblüter gelten dürfte; danach wird p-Nitrophenol zum Teil an Glucu-

1) Die Arbeit wurde mit Hilfe der Deutschen Forschungsgemeinschaft durchgeführt, wofür wir auch an dieser Stelle unseren besten Dank aussprechen. ronsäure gepaart im Harn ausgeschieden. Daneben dürfte noch eine Kupplung an Schwefelsäure und Essigsäure vorliegen $(3,4,6)$.

Für den Nachweis von p-Nitrophenol im Harn sind eine Reihe von Verfahren angegeben worden. KAISER und HAAG (2) schlagen $z$. B. den papierchromatographischen Nachweis des p-Nitrophenols und eine anschließende photometrische Auswertung vor. Fischer und KLINGELHÖLLER (7) verseifen $30 \mathrm{~m} l$ Harn mit äthanolischer Kalilauge zwei Stunden am Rückflußkühler und prüfen in dem Ätherextrakt des angesäuerten Ansatzes dünnschichtchromatographisch auf p-Nitrophenol und Diäthylthiophosphorsäure. v. EICKEN (3) hydrolysiert $1 \mathrm{ml}$ der $\mathrm{zu}$ untersuchenden Harnprobe eine Stunde im sauren Milieu, extrahiert das freigesetzte p-Nitrophenol und bestimmt es über die Indophenolblau-Reaktion. ELLIOT und Mitarbeiter (8) arbeiteten ähnlich wie v. EICKEN, setzten aber bis zu $100 \mathrm{~m} l$ Harn ein, wodurch sie noch geringere $\mathrm{p}$-Nitrophenolmengen erfassen können. Die genannten Verfahren sind sehr sicher, haben jedoch einen Zeitbedarf von einigen Stunden. Sie sind deshalb für die p-Nitrophenolbestimmung im Harn bei akuten E 605-Vergiftungen nicht ohne Modifikationen anwendbar. WeINIG hat 1956 eine 
Methode zum Nachweis von p-Nitrophenol im Harn nach Erhitzen mit Natronlauge, Extraktion im sauren Milieu und anschließender Indophenolblau-Reaktion angegeben (9):

$10 \mathrm{~m} l$ Harn werden nach kurzem Kochen mit Natronlauge mit $\mathrm{HCl}$ schwach angesäuert und mit $20 \mathrm{~m} l$ Äther extrahiert. Nach Ablassen der wäßrigen Phase werden in den Äther einige Tropfen einer wäßrigen o-Kresol-Lösung und ein linsengroßes Stück NatriumAmalgam gegeben. Am Boden des Scheidetrichters bildet sich mit eintretender alkalischer Reaktion eine leuchtend blaue Schicht aus.

Für einen Schnellnachweis erscheint es vorteilhaft, wenn nur eine sehr geringe Harnmenge einzusetzen ist (v. EICKEN) und der Zeitbedarf für die Durchführung der Reaktion gering ist (WEINIG). Wir prüften deshalb, ob in Anlehnung an diese beiden Methoden p-Nitrophenol im Harn innerhalb kurzer Zeit nachweisbar ist. Dabei sollte die Empfindlichkeit möglichst erhalten bleiben, während auf eine exakte quantitative Messung bewußt verzichtet wurde.

Nach v. ErCKen wird $1 \mathrm{~m} l$ Urin nach Verdünnung zu $10 \mathrm{~m} l$ mit $2 \mathrm{ml}$ konz. Salzsäure eine Stunde hydrolysiert. Nach dem Abkühlen werden $25 \mathrm{ml}$ eines Lösungsmittel-Gemisches (1-proz. Lösung von Isoamylalkohol in einer Mischung von $4 \mathrm{Vol}$. Petroläther und 1 Vol. Äther) zugesetzt und 10 Minuten in der Schüttelmaschine extrahiert. Nach kurzem Abzentrifugieren pipettiert man $20 \mathrm{ml}$ der Lösungsmittelschicht ab und schüttelt sie in einem $100 \mathrm{~m} /$-Scheidetrichter mit $4 \mathrm{~m} / 2 n$ Ammoniak 2 Minuten lang aus. Nach 15 Minuten läßt man die wäßrige Schicht in ein Reagenzglas ab und setzt $0,5 \mathrm{~m} l$ einer wäßrigen ges. o-Kresol-Lösung und $0,5 \mathrm{~m} l$ einer frisch bereiteten Titantrichlorid-Lösung $(3 \mathrm{ml}$ einer 15 -proz. Titantrichlorid-Lösung werden zu $50 \mathrm{~m} l$ mit dest. Wasser verdünnt) zu und schüttelt eine Minute kräftig bis zur Entfärbung der Lösung. - Nach Abzentrifugieren des Titanhydroxyds mißt man nach genau 30 Minuten die Extinktion bei $620 \mathrm{~m} \mu$ in einer $1 \mathrm{~cm}$ Küvette. Die quantitative Auswertung erfolgt an Hand einer Eichkurve. Ein p-Nitrophenolgehalt von $2,5 \mu \mathrm{g} / \mathrm{m} l \mathrm{kann}$ noch erfaßt werden.

Wir prüften, ob die von v. EICKEN angegebenen Bedingungen besonders hinsichtlich des Zeitbedarfes eingehalten werden müssen. Verwendet wurden Harne von Personen, die an einer E605-Vergiftung gestorben waren oder durch entsprechende Therapie (Atropin, PAM, künstliche Beatmung) diese überlebt hatten. Die E 605-Vergiftung wurde durch Nachweis von E 605 bzw. p-Nitrophenol in Harn, Mageninhalt, Speichel oder Organen gesichert. In einigen Fällen gelang zusätzlich auch der dünnschichtchromatographische Nachweis von Diäthylthiophosphorsäure im Harn nach Frscher und KLINGELHÖLLER (7).

In allen Harnen wurde zunächst nach der OriginalVorschrift von v. EICKEN der p-Nitrophenolgehalt bestimmt:

\begin{tabular}{ccc}
\multicolumn{3}{c}{ Tab. 1 } \\
\hline Harn-Nr. & $\begin{array}{c}\text { Extinktion des Indophe- } \\
\text { nolblaus bei } 620 \mathrm{~m} \mu\end{array}$ & $\begin{array}{c}\text { entspricht etwa } \mu \mathrm{g} \\
\text { p-Nitrophenol/m } l\end{array}$ \\
\hline 1 & 0,58 & 20 \\
2 & 1,80 & 18 \\
3 & 0,52 & 8 \\
4 & 0,03 & 19 \\
5 & 0,25 & \\
$G$ & 0,55 & \\
\hline
\end{tabular}

Für unsere Versuche verwendeten wir in der Hauptsache die Harne 1, 3 und 6, die in größerer Menge zur Verfügung standen und einen etwa gleichen p-Nitrophenol-Gehalt aufwiesen. Für die folgenden Untersuchungen wurden die in Tabelle 1 angegebenen Extinktions-Werte bei $620 \mathrm{~m} \mu$ nach Durchführung der Indophenolblau-Reaktion nach v. EICKEN als Vergleichsmaßstab benutzt, wobei diese gleich $100 \%$ gesetzt wurden.

Zur Uberprüfung des Verfahrens gingen wir im allgemeinen so vor, daß wir jeweils einen Schritt des Analysenganges variierten und im übrigen genau nach der Vorschrift arbeiteten.

\section{Hydrolyse}

Den größten Zeitbedarf bei dem p-Nitrophenol-Nachweis im Harn nach v. Eicken hat die Hydrolyse. v. Eicken (3) und Elitot (8) geben an, daß zur Bestimmung des gesamten p-Nitrophenols im Harn eine saure Hydrolyse vorgenommen werden muß, da wahrscheinlich p-Nitrophenol als Glucuronsäure-, Schwefelsäureund Essigsäure-Ester vorliegt (s. a. Williams (10)). Die genannten Autoren führen die Hydrolyse mit Salzsäure durch. v. EICKEN gibt zu $1 \mathrm{~m} l$ Harn $9 \mathrm{~m} l$ Wasser und $2 \mathrm{ml}$ konz. $\mathrm{HCl}$ und hydrolysiert eine Stunde im siedenden Wasserbad. Elurot versetzt bis zu $100 \mathrm{~m} l$ Harn mit $20 \mathrm{ml}$ konz. $\mathrm{HCl}$ und erhitzt gleichfalls eine Stunde im kochenden Wasserbad. Die Säurekonzentration liegt also bei beiden Autoren etwa gleich. Elliot gibt an, daß durch Verlängerung der Hydrolysezeit bis auf vier Stunden oder durch Erhöhung der Säuremenge keine Verbesserung der p-Nitrophenol-Ausbeute zu erreichen war. Wir versuchten trotzdem, durch höhere SäureKonzentration Hydrolysezeit einzusparen.

\section{Hydrolyse mit Salqsäure}

Hydrolysebedingungen nach v. ErCKEN bei Änderung der Hydrolysezeiten:

Unter alleiniger Veränderung der Hydrolysezeit wurden die Harne 3 und 6, sowie zum Vergleich zwei p-Nitrophenol-freie Harne $\left(\mathrm{N}_{1}\right.$ und $\left.\mathrm{N}_{2}\right)$ genau nach v. EICKEN untersucht. Tabelle 2 zeigt die nach verschiedener Erhitzungsdauer erhaltenen Werte:

Tab. 2

\begin{tabular}{cccc}
\hline $\begin{array}{c}\text { Erhitzungsdauer in } \\
\text { Minuten }\end{array}$ & \multicolumn{3}{c}{ Extinktion der Harne Nr. } \\
\hline 120 & 3 & 6 & 6 \\
60 & 0,62 & 0,66 & 0,63 \\
45 & 0,52 & 0,55 & 0,54 \\
30 & 0,50 & 0,52 & 0,51 \\
15 & 0,47 & 0,50 & 0,50 \\
& 0,44 & 0,40 & 0,41 \\
\hline
\end{tabular}

Daraus ergibt sich, daß die Indophenolblau-Färbung wohl durch Freisetzung einer größeren p-Nitrophenolmenge - nach länger dauernder Hydrolyse intensiver ist. Die beiden p-Nitrophenolfreien Normalharne brachten stets ein negatives Ergebnis. 
Hydrolyseverlauf bei höherer $\mathrm{HCl}-$ Konzentration und Variation der Hydrolysezeit:

Je $1 \mathrm{~m} /$ der Harne 1, 3 und 6 wurden mit $8 \mathrm{~m} /$ konz. $\mathrm{HCl}$ und $3 \mathrm{~m} l \mathrm{H}_{2} \mathrm{O}$ versetzt und $5,15,30$ und 60 Minuten lang im verschlossenen Glaskolben im siedenden Wasserbad erhitzt. Nach Beendigung der Hydrolyse wurde nach v. EICKEN weitergearbeitet. Die beiden Normalharne zeigten unter diesen Bedingungen keinerlei Blaufärbung. Bei Harn 3 wurde schon nach 13 Minuten Erhitzungsdauer der gleiche Extinktionswert wie unter den v. EICKENschen Bedingungen nach 60 Minuten erhalten, während dies bei den Harnen Nr. 1 und 6 etwa 25 Minuten beanspruchte. Das verschiedene Verhalten der Harne kann daher rühren, daß p-Nitrophenol in unterschiedlichem Verhältnis an Glucuron-, Schwefel- und Essigsäure gebunden ist. Bei allen drei Harnen aber erwies es sich, daß durch eine Erhöhung der Säurekonzentration Hydrolysezeit einzusparen ist.

\section{Hydrolyse mit Perclblorsäure}

Perchlorsäure eignet sich nicht zur Hydrolyse, da hier auch in den Normalharnen nach Durchführung der Indophenolblau-Reaktion grüne bis blaue Verfärbungen auftraten, die bei der spektrophotometrischen Untersuchung Maxima bei 620 und $630 \mathrm{~m} \mu$ aufwiesen.

\section{Hydrolyse mit Scbıvefelsäure}

Harn Nr. 6 wurde nach v. EICKEN aufgearbeitet, mit dem Unterschied, daß an Stelle von $2 \mathrm{~m} l$ konz. $\mathrm{HCl} 2 \mathrm{~m} l$ konz. Schwefelsäure verwendet wurden. Die Extinktion bei $620 \mathrm{~m} \mu$ stieg hierdurch von 0,55 auf 0,65 . Es war also bereits eine Empfindlichkeitssteigerung gegenüber dem Vorgehen nach v. EICKEN eingetreten.

Um die günstigsten Bedingungen einer Hydrolyse mit Schwefelsäure zu ermitteln, hydrolysierten wir Harn Nr. 6 mit wechselnden Schwefelsäurekonzentrationen und bei verschiedenen Hydrolysezeiten, wobei die Gesamtmenge des Hydrolyse-Ansatzes immer $1 \mathrm{ml}$ Harn in $12 \mathrm{~m} l$ Gesamtvolumen betrug. Zunächst wurden die einzelnen Hydrolyse-Gemische 10 bis 15 Sekunden lang im offenen Glaskolben über der Flamme bis zum Entstehen der ersten Rauchschwaden aufgekocht, außerdem wurde im verschlossenen Glaskolben 15, 30, 45 und $60 \mathrm{Mi}-$ nuten lang im siedenden Wasserbad erhitzt. Nach dem Abkühlen arbeiteten wir genau nach v. EICKEN weiter. Die besten Ergebnisse wurden mit einem HydrolyseAnsatz aus $1 \mathrm{~m} / \mathrm{Harn}, 7 \mathrm{~m} / \mathrm{H}_{2} \mathrm{O}$ und $4 \mathrm{~m} / \mathrm{konz} . \mathrm{H}_{2} \mathrm{SO}_{4}$ erreicht. Damit war schon beim kurzen Aufkochen über der Flamme eine um etwa $40 \%$ bessere Extinktion als mit der Original-Methode zu erzielen. Hiermit ergibt sich eine Zeitersparung von praktisch einer Stunde. Längeres Erhitzen bei hoher Schwefelsäure-Konzentration führt offenbar zu einer Zerștörung des p-Nitrophenols, da die Extinktionswerte wieder abnahmen.

Mit den Harnen Nr. 1, 2, 3, 4, 5 und 6 wurde beim gleichen Vorgehen ebenfalls ein Indophenolblau mit einem Absorptionsmaximum bei $620 \mathrm{~m} \mu$ erhalten, wobei die Extinktionen etwa 10 bis $20 \%$ über den nach der Originalvorschrift erhaltenen lagen. 10 in der gleichen
Weise aufgearbeitete E 605-freie Normal-Harne wiesen entweder keine Färbung auf oder zeigten nur eine leichte hellbraune Verfärbung.

\section{Hydrolyse in alkalischen Milieu}

WeINIG hydrolysiert die Harnproben durch Kochen mit Natronlauge, machte in seiner Diskussionsbemerkung jedoch keine näheren Angaben über Erhitzungsdauer und verwendete Natronlauge-Konzentration. Deswegen prüften wir vergleichend auch die Hydrolyse-Bedingungen und Resultate im alkalischen Milieu:

Je $1 \mathrm{~m} l$ der Harne 1,2 und 3 wurden mit $8 \mathrm{~m} l 2 n \mathrm{Na}$ tronlauge versetzt und eine Viertelstunde im siedenden Wasserbad erhitzt. Sodann wurde stark angesäuert und nach v. EICKEN weitergearbeitet. In allen drei Fällen trat nach Durchführung der' Indophenolblau-Reaktion nur eine ganz leichte Blaufärbung auf. Daraus ist zu schließen, $\mathrm{da} ß$ unter diesen Bedingungen gepaartes p-Nitrophenol nur in geringem Umfange gespalten wird. Wir führten dann Versuche mit Erhöhung der Laugen-Konzentration und wechselnder Erhitzungsdauer durch, wobei wir je $1 \mathrm{ml}$ Harn einsetzten und die Gesamtmenge des HydrolyseGemisches wieder auf $9 \mathrm{~m} l$ brachten. Es zeigte sich, daß selbst bei Verwendung einer hohen Natronlauge-Konzentration ( $1 \mathrm{~m} l$ Harn, $8 \mathrm{~m} l 4 n \mathrm{NaOH})$ und einer Hydrolysezeit von 60 Minuten nur etwa $1 / 3$ der nach v. ErCKEN erhaltenen Extinktion zu erreichen war.

Extraktion mit organischem Lösungsmittelgemisch

\section{Lösungsmittelgemisch}

Wie Lawford und Harvey wendet auch v. EICKEN zur Extraktion des p-Nitrophenols aus dem hydrolysierten Harn eine 1-proz. Lösung von Isoamylalkohol in einer Mischung aus 4 Vol. Petroläther und 1 Vol. Äther an. Elcror (8) extrahiert mit einem Gemisch aus Äthyläther und Benzol im Verhältnis 20:80.

Wir untersuchten zunächst die Harne 3 und 6 je dreimal nach v. EiCKEN, wobei lediglich an Stelle von $25 \mathrm{~m} /$ des v. EICKENschen Lösungsmittelgemisches $25 \mathrm{~m} l$ des Lösungsmittelgemisches nach ElLIOT verwendet wurden. Mit beiden Lösungsmittelgemischen wurden völlig übereinstimmende Ergebnisse erzielt, deshalb verwendeten wir für unsere weiteren Untersuchungen wie v. ErCKEN als Extraktionsmittel Isoamylalkohol-Äther-Petroläther.

\section{Extraktionsdauer}

v. EICKEN extrahiert das durch Hydrolyse freigesetzte pNitrophenol mit $25 \mathrm{~m} l$ des Lösungsmittelgemisches aus der sauren wäßrigen Phase durch 10 Minuten langes Schütteln.

Wir führten zunächst Versuche mit einer p-Nitrophenol-Lösung durch, die $7 \mu \mathrm{g}$ p-Nitrophenol pro $\mathrm{m} l$ enthielt. Zu je $1 \mathrm{~m} l$ dieser Lösung wurden $2 \mathrm{~m} l$ konz. Salzsäure, $9 \mathrm{~m} l \mathrm{H}_{2} \mathrm{O}$ und $25 \mathrm{~m} l$ des v. Eickenschen Lösungsmittelgemisches in einen Scheidetrichter gegeben. Darauf wurde $1 / 4$ bis 10 Minuten lang geschüttelt, der Scheidetrichter 15 Minuten ruhig stehen gelassen und dann die wäßrige Phase abgetrennt; weiterer Arbeitsgang 
nach v. EICKEN. Es zeigte sich, daß nach 15 Sekunden Schütteln schon $2 / 3$ des p-Nitrophenols in das Lösungsmittelgemisch übergegangen waren. Nach 30 Sekunden Schütteln ergaben sich schon die gleichen Extinktionswerte wie nach 10 Minuten dauernder Extraktion. Nach diesem Vorversuch hydrolysierten wir die Harne 3 und 6 nach v. Ercken, extrahierten jedoch nur eine Minute mit dem organischen Lösungsmittelgemisch, und gingen dann weiter nach v. ErCKEN vor. Dabei ergaben sich die gleichen Extinktionen wie nach der Originalmethode.

\section{Phasentrennung nach Extraktion}

v. EICKen zentrifugiert nach Extraktion mit dem Lösungsmittelgemisch, um die wäßrige von der organischen Phase zu trennen. Wir stellten fest, daß ein Zentrifugieren nicht erforderlich ist, $\mathrm{da}$ sich nach vier $\mathrm{Mi}$ nuten langem Stehen im Scheidetrichter die Phasen praktisch immer vollständig voneinander getrennt hatten.

\section{Extraktion mit $2 n$ Ammoniak}

v. EICKEN schüttelt p-Nitrophenol aus dem organischen Lösungsmittelgemisch mit $2 n \mathrm{NH}_{4} \mathrm{OH}$ während zwei Minuten aus. Wir suchten auch diesen Teilvorgang $z u$ verkürzen und stellten sowohl mit der oben beschriebenen p-Nitrophenol-Lösung ( $1 \mathrm{~m} l$ p-Nitrophenol, $2 \mathrm{~m} l$ $\mathrm{HCl}, 9 \mathrm{~m} l \mathrm{H}_{2} \mathrm{O}$ ) wie auch mit je $1 \mathrm{ml}$ der Harne Nr. 3 und 6 fest, daß eine Schütteldauer von 1 Minute ausreichend ist.

Phasentrennung nach Extraktion mit $2 n \mathrm{NH}_{4} \mathrm{OH}$ Nach der Extraktion mit $2 n$ Ammoniak läßt v. EICKEN den Scheidetrichter 15 Minuten stehen, um die organische von der wäßrigen Phase zu trennen. Nach unserer Beobachtung (p-Nitrophenollösung und Harne 3 und 6) genügen 5-8 Minuten, um eine gute Trennung zu erreichen.

\section{Reduktionsmittel}

Als Reduktionsmittel zur Umwandlung des p-Nitrophenols in p-Aminophenol wählt WeINIG NatriumAmalgam, v. Ercken Titantrichlorid. Um diese beiden Reduktionsmittel in ihrer Wirksamkeit zu vergleichen, verwendeten wir im Analysengang nach v. EICKEN zur Reduktion die von WeINIG angegebene Menge NatriumAmalgam. Es zeigte sich, daß der Indophenolblau-Farbstoff wesentlich langsamer gebildet wird und sich nicht so intensiv entwickelt wie mit Titantrichlorid. Deshalb ist -Natrium-Amalgam für einen Schnellnachweis weniger geeignet.

\section{Bildungsgeschwindigkeit des Indophenol}

Um die nach Zusatz von Titantrichlorid und o-Kresol bis zur vollen Ausbildung des Indophenol-Farbstoffes erforderliche Zeit festzustellen, die bei v. EICKEN mit 30 Minuten angegeben wird, prüften wir mit Harn Nr. 1 nach v. ErCKeN auf p-Nitrophenol und variierten die Zeit vom Zusatz des o-Kresol und Titantrichlorid bis zur Messung von 5 bis 60 Minuten. Es zeigte sich, daß die Farbe schon nach 5 Minuten praktisch voll ausge- bildet war und während 60 Minuten kein Abfall der Extinktionswerte auftrat. Es ist also nicht unbedingt erforderlich, bis zur Messung der größten Farbtiefe 30 Minuten $\mathrm{zu}$ warten. Eine Erhöhung der Titantrichlorid-Menge auf das 5 fache erbrachte keine schnellere Ausbildung der maximalen Farbintenșität.

Wahl einer anderen Kondensationskomponente v. ErCKEN und ElLrot verwenden zur IndophenolblauReaktion als Kondensationskomponente o-Kresol in wäßriger Lösung. Schon von WALDMANN und KROUSE, sowie Mountan, Zlotolow und O'Conor wurde Phenol an Stelle des o-Kresol vorgeschlagen. W. Pilz (11) stellte durch vergleichende Untersuchungen fest, daß dem unsubstituierten Phenol vor p-Kresol, o-Kresol, 2,6Diäthylphenol (1), 2,5-Dimethyl-phenol (1) und 3-Methyl-5-Äthylphenol (1) der Vorzug zu geben ist, weil es die größte Farbintensität ergibt. Mit Phenol liegt das Maximum der Absorptionskurve des Indophenolfarbstoffes bei $629 \mathrm{~m} \mu$. Deshalb wurde bei den nachfolgenden Versuchen mit Phenol die Messung bei $629 \mathrm{~m} \mu$ durchgeführt. Wir ersetzten in der Versuchsanordnung nach v. ErCKen (p-Nitrophenol-Lösung, Harne 3 und 6) die o-Kresol-Lösung durch die gleiche Menge einer Phenol-Lösung, die 1, 2 oder $10 \mathrm{~g}$ Phenol in $10 \mathrm{~m} / \mathrm{H}_{2} \mathrm{O}$ enthielt. Es zeigte sich, daß durch Ersatz des o-Kresols durch Phenol die gemessenen Extinktionen um 25\% höher lagen, selbst wenn schon 5 Minuten nach Zugabe von Titantrichlorid und Phenol gemessen wurde. Bei Verwendung von Phenol ist allerdings mit einer weiteren Zunahme der Extinktion zu rechnen, da sich nach Pilz die volle Farbtiefe erst nach 4,5 Stunden ausbildet. Als geeignetes Reagenz erwies sich eine Lösung von $2 \mathrm{~g}$ Phenol in $10 \mathrm{~m} l$ Wasser.

\section{Arbeitsvorschrift und Diskussion}

Fą̧ man die gewonnenen Ergebnisse zusammen, so ist es möglich, durch Änderung der Bedingungen für den p=Nitrophenolnachweis im Harn nach v. EICKEN einen Schnellnachweis für $\mathrm{p}-\mathrm{Nitrophenol} \mathrm{im} \mathrm{Harn} \mathrm{anzugeben.}$ Die Arbeitsvorschrift lautet:

$1 \mathrm{~m} l \mathrm{Harn}, 7 \mathrm{~m} l \mathrm{H}_{2} \mathrm{O}$ und $4 \mathrm{ml}$ konz. Schwefelsäure werden im offenen Erlenmeyerkolben auf einem Drahtnetz bis zum Auftreten der ersten Rauchschwaden etwa 15 Sek. lang gekocht. Nach dem Abkühlen werden $25 \mathrm{ml}$ des Lösungsmittelgemisches (1-proz. Lösung von Isoamylalkohol in einer Mischung von $4 \mathrm{Vol}$. Petroläther und 1 Vol. Äther) in einem $100 \mathrm{~m} l$ Scheidetrichter dem Hydrolyse-Gemisch zugesetzt. Sodann wird 1 Minute geschüttelt. Vier Minuten später läßt man die wäßrige Phase ab und schüttelt die organische Phase in einem neuen, sauberen Scheidetrichter mit $4 \mathrm{~m} / 2 n$ Ammoniak eine Minute lang aus. Nach der Phasentrennung (5 Minuten) läßt man die wäßrige Schicht in ein Reagenzglas ab und setzt $0,5 \mathrm{ml}$ einer farblosen wäßrigen Phenol-Lösung $(2 \mathrm{~g}$ Phenol in $10 \mathrm{~m} l \mathrm{H}_{2} \mathrm{O}$ ) hinzu. (Eine schon jetzt auftretende Blaufärbung kann nicht auf p-Nitrophenol zurückgeführt werden. Sie deutet auf die Anwesenheit von Aminophenolen, z. B. nach Phenacetinaufnahme hin.) Sodann gibt $\operatorname{man} 0,5 \mathrm{ml}$ einer frisch angesetzten wäßrigen Titantrichlorid-Lösung ( $0,06 \mathrm{ml} 15$-proz. Titantrichlorid pro $\mathrm{m} / \mathrm{H}_{2} \mathrm{O}$ ) zu und schüttelt eine halbe Minute kräftig bis zur Entfärbung der Lösung. Der blaue Indophenol-Farbstoff ist bei Anwesenheit von mindestens $2 \mu \mathrm{g}$-Nitrophenol $/ \mathrm{m} / \mathrm{Harn}$ nach 5 Minuten deutlich zu erkennen. 
Nach dieser Vorschrift sind für den Nachweis insgesamt etwa 15 Minuten erforderlich. Mit seiner Hilfe kann in vielen Fällen schnell die Aufnahme von E 605 wahrscheinlich gemacht werden. Selbstverständlich ist daran zu denken, daß gelegentlich auch andere Substanzen, wie z. B. Phenacetin- oder Anilin-Abbauprodukte eine positive Indophenolblau-Reaktion im Harn ergeben kön- nen $(2,8)$ und daß p-Nitrophenole auch als Metaboliten anderer Verbindungen wie z. B. Chlorthion ausgeschieden werden können. Daneben schließt ein negativer Ausfall des Nachweises eine E 605-Vergiftung nicht aus, da bei sehr raschem Verlauf kein p-Nitrophenol mehr im Harn erscheint.

\title{
Literatur
}

1. SCHWERd, W. u. G.Schmidt, Dtsch. med. Wschr. 77, 372 (1952). - 2. Kaiser, H. und Th. HaAg, Arch. Pharmaz. 289/61, 542 (1956). - 3. v. Ercken, S., Angew. Chem., Ausg. A. 66, 551 (1954). - 4. Mountain, J. T., H. Zlotolow and D. G. Harvey, Indian Health Monthly 11, 88 (1950). - 5. O'BrIEN, R. D., Toxic phosphorous esters, Academic Press, New York-London (1960). 6. ErdmanN, W. D. und L. Lendle, Erg. inn. Med. 10, 104 (1958).
- 7. Fischer, R. und W. Klingelhöller, Arch. Toxikol. 19, 119 (1961). - 8. Elliot, J. W., K. G. Walker, A. E. Penick und W. F. Durhim, J. agric. Food Chem. 8, 111 (1960). - 9. Weinig, E., Dtsch. Zschr. gerichtl. Med. 46, 269 (1957). - 10. Williams, R. T., Detoxication mechanism, S. 136, Chapman \& Hall, London (1959). - 11. Przz, W., Microchim. Acta (Wien), 383 (1958).

Priv.-Doz. Dr. Dr. Marika Geldmacher-v. Mallinckrodt Inst. für gerichtl. Medizin und Kriminalistik der Universität Erlangen-Nürnberg

852 Erlangen, Universitätsstr. 22

\section{KURZMITTEILUNG}

\section{Einfacher Entfärbeapparat für die Disk-Elektrophorese}

\author{
Von H. R. MAURER \\ Aus der Division of Biology, California Institute of Technology, Pasadena, California, US A
}

(Eingegangen am 19. Januar 1966)

In letzter Zeit gewinnt die Disk-Elektrophorese (1), eine neuere, analytische Methode zur Trennung, Darstellung und Reinheitsprüfung von Proteinen und anderen hochmolekularen Polyelektrolyten, zunehmend an Bedeutung, weil sie sich als wesentlich empfindlicher und zumeist auch reproduzierbarer als Papier- und StärkegelElektrophorese erweist. Weitere Vorteile liegen in der Vielseitigkeit hinsichtlich der Trennmöglichkeit von Proteinen verschiedenster Größe und Gestalt, im kurzen Zeitaufwand und für die Auswertung in der Transparenz der Polyacrylamidgele. Wenn auch zur Zeit noch keine statistisch gesicherten Reihenuntersuchungen größeren Ausmaßes vorliegen, so sind doch die bislang gewonnenen Ergebnisse bei der Diagnose von Makroglobulinämie (2,7), Differentialdiagnose von multiplem Myelom und Waldenströmscher Makroglobulinämie $(7,10)$, Paraproteinämie (3), Plasmozytom $(2,4,7)$, zystischer Pankreasfibrose (5), rheumatischem Fieber mit Pankarditis (6), Tuberkulose (6), genuiner Pneumonie (6), Polyneuritis $(8,9)$, u. a. vielversprechend.

Zum Sichtbarmachen der mittels Disk-Elektrophorese getrennten Proteine werden die Gele zumeist mit Amido-Schwarz gefärbt. Entfärben kaṇn man durch erschöpfendes Auswaschen des Farbüberschusses oder durch Elektrophorese des ungebundenen Farbstoffes.
Vor- und Nachteile beider Methoden sind in Tabelle 1 zusammengefaßt.

Tab. 1

Vergleich der Entfärbemethoden

\begin{tabular}{|c|c|c|}
\hline Methode & Vorteile & Nachteile \\
\hline $\begin{array}{l}\text { Einfaches Aus- } \\
\text { waschen (durch } \\
\text { Diffusion) }\end{array}$ & $\begin{array}{l}\text { Erhaltung schwach } \\
\text { fixierter Proteine }\end{array}$ & $\begin{array}{l}\text { Zeitaufwand; Unter- } \\
\text { grundfärbung nicht } \\
\text { immer entfernbar, da- } \\
\text { durch Erschwerung } \\
\text { der densitometrischen } \\
\text { Auswertung }\end{array}$ \\
\hline $\begin{array}{l}\text { Elektrophorese des } \\
\text { ungebundenen } \\
\text { Farbstoffes }\end{array}$ & $\begin{array}{l}\text { Rasche, voll- } \\
\text { kommene Ent- } \\
\text { färbung } \\
(20-40 \mathrm{Min} .)\end{array}$ & $\begin{array}{l}\text { Verlust schwach fi- } \\
\text { xierter Proteine; gra- } \\
\text { duelle Abnahme der } \\
\text { Farbintensität und } \\
\text { Erhöhung des elektr. } \\
\text { Widerstandes durch } \\
\text { Gasblasenbildung } \\
\text { möglich }\end{array}$ \\
\hline
\end{tabular}

Systematische Untersuchungen mit basischen Proteinen relativ kleinen Molekulargewichtes (Histonen) haben ergeben, daß zur genügenden Färbung und Fixierung in 1-proz. Amidoschwarzlösung (20\% Äthanol, 7\% Eis- 